\title{
Experimental evaluation of absorption coefficient of insulated vehicle sandwich panel.
}

\author{
by P. Bison*, A. Dragano* and S. Rossi*
}

*Construction Technologies Institute - National Research Council, Padua, Italy

\begin{abstract}
ATP is an international agreement among European States concerning transport of perishable foodstuffs and special equipments to be used for such transport. The purpose of the ATP is to improve the conditions of preservation of the quality of foodstuffs during their carriage, especially regarding quick-frozen and deep-frozen food, in order to keep safe the cold-chain. The aim of this work is to evaluate the absorption coefficient of the sandwich panel that is the basis of the standard insulated vehicle used in refrigerated transport. The evaluation of this coefficient is useful in the complex study of thermal balance of the vehicle.
\end{abstract}

\section{Introduction}

The main purpose of the study is to determine the absorption coefficient of the sandwich panel, consisting of GFRP (Glass Fiber Reinforced Plastic), polyurethane and GFRP, by the use of IR thermocamera. The mathematical model is based on the analytical solution of heat transfer in a semi-infinite two-layer surface consisting of a finite first layer and a semi-infinite second layer.

\section{Mathematical Model}

It's been considered a semi-infinite two-layer surface with the following boundary conditions:

Constant thermal flux (Heaviside step function) and even on the surface $(x=0)$

Adiabatic heat transfer with the external system

Negligible initial temperature of the panel

Homogeneous and isotropic heat transfer model

The following Eq. (1) is the temperature function along time (analytical solution of heat transfer in the above conditions):

$$
T_{x=0}(t)=\frac{2 F_{0}}{e_{c}} \sqrt{\frac{t}{\pi}}\left\{1+2 \sum_{n=1}^{\infty} \Gamma^{n}\left[e^{-\frac{n^{2} l_{c}^{2}}{\alpha_{c} t}}-\sqrt{\frac{\pi n^{2} l_{c}^{2}}{\alpha_{c} t}} \operatorname{erfc}\left(\sqrt{\frac{n^{2} l_{c}^{2}}{\alpha_{c} t}}\right)\right]\right\}
$$

where:

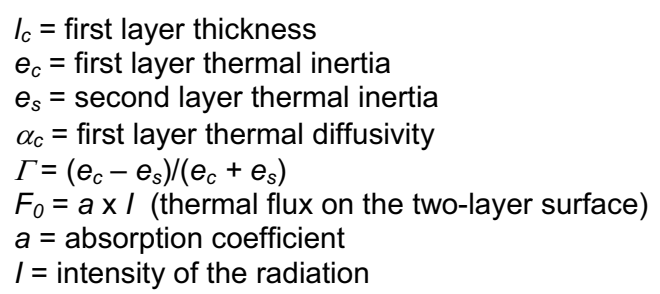

\section{Experimental Appliances}

The focus of the experiments is a sandwich panel of GFRP + polyurethane + GFRP: it measures approximately $1000 \times 1000 \mathrm{~mm}$ and its thickness is $80 \mathrm{~mm}(1,5 \mathrm{~mm}$ GFRP $+77 \mathrm{~mm}$ polyurethane $+1,5 \mathrm{~mm}$ GFRP); in the centre of the panel two symmetrical target spots (100 mm diameter and $200 \mathrm{~mm}$ relative distance) have been marked: the first is painted in black (absorption coefficient assumed to be equal to 1) and the second hasn't been modified (this is the zone of the unknown absorption coefficient).

The panel has been set vertically (Figure 1) and in front of it has been placed a tungsten lamp (1000 W) in order to heat up the surface of the panel; near the lamp has been placed an infrared thermocamera to get the surface temperature. The relative position of the lamp and thermocamera has been changed during various tests.

The experimental conditions have been modified to be as near as possible to the theory; for example, to get the hypothesis of semi-infinite surface, the two target zones have been placed in the centre of an extended 
surface and the data collection (temperatures of white and black spots) is limited to a finite period, much more short of the period of propagation of thermal flux inside the panel thickness.

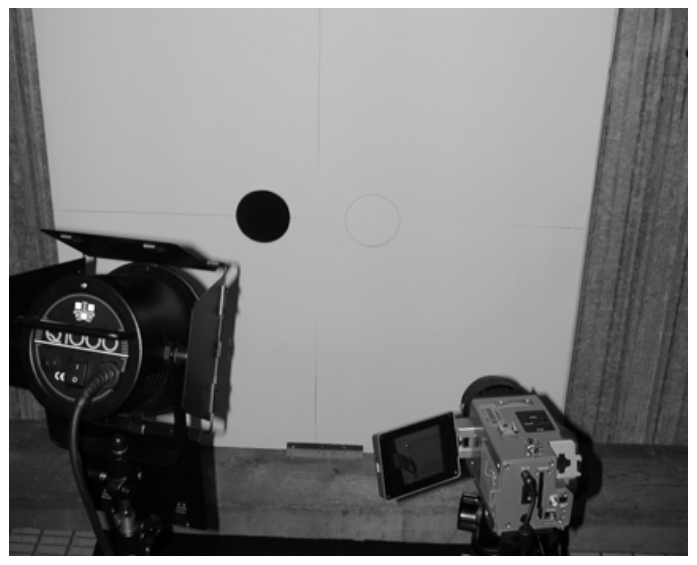

Fig. 1. Experimental Appliances

\section{Data measurements}

In the above conditions, the temperatures of "white" spot and black spot have been measured by infrared thermocamera and the typical graph of temperature versus time (square root) is represented below (figure 2).

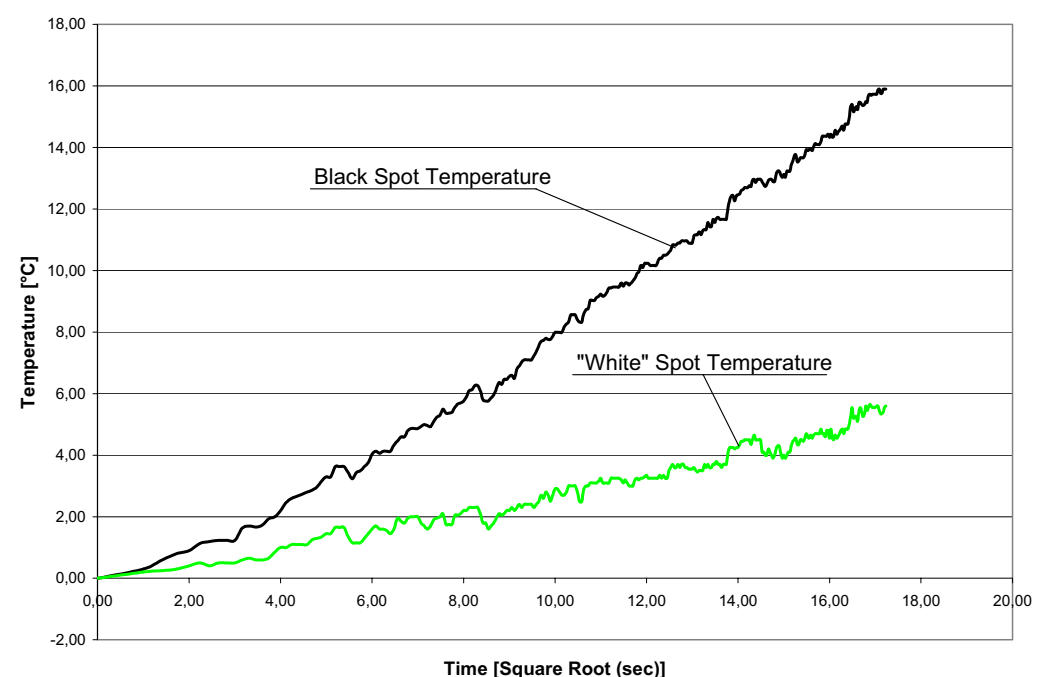

Fig. 2. Temperatures of black and white spots

From the figure it's possible to notice two different parts in the curve of temperatures: a first period (of about 25 seconds) and a second period where the slope of the curve changes; the change of the slope is due to the passage of thermal flux from the first layer in GRFP to the second layer (polyurethane) and this slope is wellfitted by theoretical equation of two-layer (Eq. 1).

Because of the type of lighting and the fact that it could not be considered like solar radiation (beam of parallel rays), it's been decided to invert the two target spots during the experimental tests: so two absorption coefficients were obtained, $a^{\prime}$ and $a^{\prime \prime}$, and the final coefficient is equal to their square root (Eq. 2).

$$
a=\sqrt{\frac{T_{W}^{\mathrm{I}}}{T_{B}^{\mathrm{I}}} \frac{T_{W}^{\mathrm{II}}}{T_{B}^{\mathrm{II}}}}=\sqrt{a^{\mathrm{I}} a^{\mathrm{II}}}
$$

